\title{
THE DUAL OF A GÂTEAUX SMOOTH BANACH SPACE IS WEAK STAR FRAGMENTABLE
}

\author{
N. K. RIBARSKA
}

(Communicated by William J. Davis)

\begin{abstract}
The techniques of Preiss-Phelps-Namioka is used to prove that if a Banach space $E$ admits a Gâteaux smooth norm then its dual $E^{*}$, endowed the weak star topology, is fragmentable.
\end{abstract}

\section{INTRODUCTION}

The main aim of this paper is to prove the title statement and to establish further relations between the theory of weak Asplund spaces and the notion of fragmentability. The latter notion was introduced by Jayne and Rogers in [JR].

0.1 . Definition. Let $X$ be a topological space and $\rho$ be a metric on it. We say that $\rho$ fragments $X$ if for every $\varepsilon>0$ and for every nonempty subset $Y$ of $X$ there exists a nonempty relatively open subset $U$ of $Y$ whose $\rho$-diameter is not greater than $\varepsilon$. The topological space $X$ is said to be fragmentable if there exists a fragmenting metric on it.

0.2. Definition. The Banach space $E$ is called weak Asplund if every convex function defined on an open convex subset of $E$ is Gâteaux differentiable at each point of a dense $G_{\delta}$ subset of its domain.

For additional information about weak Asplund spaces the reader is referred to the book of R. Phelps [Ph].

0.3. Theorem of Asplund. If the dual space $E^{*}$ admits a dual strictly convex norm then $E$ is weak Asplund.

Since, in this case, the corresponding norm in $E$ is Gâteaux differentiable (off zero), it was natural to ask if the assertion of the above theorem of Asplund is valid for every Banach space $E$ with a norm which is differentiable at each nonzero point (i.e., a Gâteaux smooth norm). This long standing question was recently answered positively in [PPhN]:

0.4. Theorem of Preiss-Phelps-Namioka. If the norm of $E$ is Gâteaux smooth then $E$ is weak Asplund.

Received by the editors August 12, 1990.

1991 Mathematics Subject Classification. Primary 46B20, 54C60.

Key words and phrases. Weak Asplund spaces, fragmentability. 
The connection between the notions "fragmentability" and "weak Asplund" is revealed by the following statement, which was proved in [ChK].

0.5. Theorem. If $E^{*}$ with its weak-star topology is fragmentable then $E$ is weak Asplund.

It was not clear, however, whether this result implies the above mentioned theorems of Asplund and Preiss-Phelps-Namioka, respectively. In [R1] the author has proved that this is the case for the theorem of Asplund. Combining this with the techniques developed in $[\mathrm{PPhN}]$ we show in $\S 1$ that the PreissPhelps-Namioka theorem can also be considered as a corollary of Theorem 0.5. (In fact, our main result is a further step after Theorems 1.6 and 2.2 of [PPhN] and its proof uses the same construction.)

Our approach needs no "external notions" like games and mappings. It provides additional information about the intrinsic structure of $\left(E^{*}, w^{*}\right)$.

The results in the last section generalize the theorems from $\S 3$ of $[\mathrm{PPhN}]$.

The proofs in this paper make use of a characterization of fragmentable spaces by the following notion.

0.6. Definition. A well-ordered family $\mathfrak{U}=\left\{U_{\xi}: 0 \leq \xi<\xi_{0}\right\}$ of subsets of the topological space $X$ is said to be a relatively open partitioning of $X$ if

(i) $U_{0}=\varnothing$;

(ii) $U_{\xi}$ is contained in $X \backslash\left(\bigcup_{\eta<\xi} U_{\eta}\right)$ and is relatively open in it for every $\xi, 0<\xi<\xi_{0}$;

(iii) $X=\left(\bigcup_{\xi<\xi_{0}} U_{\xi}\right)$.

A family $\mathfrak{U}$ of subsets of the topological space $X$ is said to be a $\sigma$-relatively open partitioning of $X$ if $\mathfrak{U}=\bigcup_{n=1}^{\infty} \mathfrak{U}^{n}$, where $\mathfrak{U}^{n}, n=1,2, \ldots$ are relatively open partitionings of $X . \mathfrak{U}$ is said to separate the points of $X$ if whenever $x$ and $y$ are two different elements of $X$ there exists $n$ such that $x$ and $y$ belong to different elements of the partitioning $\mathfrak{U}^{n}$. In this case we say that $X$ admits a separating $\sigma$-relatively open partitioning.

A topological space $X$ is fragmentable if and only if it admits a separating $\sigma$-relatively open partitioning (see [R, Theorem 1.9]).

\section{Duals of Gâteaux Smooth SPaces}

1.1. Theorem. Let $E$ be a Banach space and $\|\cdot\|$ be a Gâteaux smooth norm on it. Then the topological space $\left(E^{*}, w^{*}\right)$ is fragmentable.

Proof. Step 1. Let us denote by $B^{*}$ the dual unit ball. As $E^{*}=\bigcup_{m=1}^{\infty} m B^{*}$, it suffices to prove that $\left(B^{*}, w^{*}\right)$ is a fragmentable space.

Step 2. Basic step of the construction.

Let $U$ be a subset of $B^{*}, p$ be an equivalent norm on $E$, and $\varepsilon, \beta$ be two positive numbers. Then we construct a relatively open partitioning $\mathfrak{B}=\left\{V_{\xi}\right.$ : $\left.0 \leq \xi<\xi_{0}\right\}$ of $\left(U, w^{*}\right)$ and associate to every element $V$ of $\mathfrak{B}$ a nonnegative real number $s_{V}$, an element $e_{V}$ of $E$ with $p\left(e_{V}\right)=1$, and a new equivalent norm $p_{V}$ on $E$ in the following way.

We put $V_{0}=\varnothing, s_{V_{0}}=0$, and $e_{V_{0}}, p_{V_{0}}$ be arbitrary.

If we have constructed $V_{\eta}$ for every ordinal number $\eta<\xi$, we consider the set $R_{\xi}=U \backslash\left(\bigcup_{\eta<\xi} V_{\eta}\right)$. If $R_{\xi}=\varnothing$, we put $\xi_{0}=\xi$ and finish the process. 
If not, we put $s_{V_{\xi}}=\sup \left\{p^{*}\left(x^{*}\right): x^{*} \in R_{\xi}\right\}$ where $p^{*}$ is the norm conjugate to $p$.

If $s_{V_{\xi}}=0$, it means that $R_{\xi}=\{0\}$. Then we put $V_{\xi}=\{0\}, \xi_{0}=\xi+1$, and $e_{V_{\xi}}, p_{V_{\xi}}$ be arbitrary and finish again. set

If $s_{V_{\xi}}>0$, there exists an element $e_{V_{\xi}}$ of $E$ with $p\left(e_{V_{\xi}}\right)=1$ such that the

$$
V_{\xi}=\left\{x^{*} \in R_{\xi}:\left\langle x^{*}, e_{V_{\xi}}\right\rangle>(1-\varepsilon) s_{V_{\xi}}\right\}
$$

is nonempty. Moreover, $V_{\xi}$ is $w^{*}$-relatively open in $R_{\xi}$. We put $q_{V_{\xi}}(x)=$ $\inf \left\{\left\|x-\lambda e_{V_{\xi}}\right\|: \lambda \in \mathbb{R}\right\}$ for every $x \in E$ and $p_{V_{\xi}}^{2}=p^{2}+\beta \cdot q_{V_{\xi}}$. Thus the relatively open partitioning $\mathfrak{B}$ is constructed.

Step 3. Construction of the $\sigma$-relatively open partitioning $\mathfrak{U}=\bigcup_{n=1}^{\infty} \mathfrak{U}^{n}$ of $\left.\left(B^{*}, w^{*}\right)\right)$. Following the construction in [PPhN] we fix two sequences of positive numbers $1 / 2>\varepsilon_{1}>\varepsilon_{2}>\cdots$ and $\beta_{1}>\beta_{2}>\cdots$ such that $\varepsilon_{k} \rightarrow 0$, $\sum \beta_{k}^{2}<3$, and $\sum\left(\varepsilon_{k}\right)^{1 / 2} / \beta_{k}<\infty$.

Applying the basic step with $U:=B^{*}, p:=\|\cdot\|, \varepsilon:=\varepsilon_{1}$, and $\beta:=\beta_{1}$, we obtain a relatively open partitioning $\mathfrak{U}^{1}=\left\{U_{\xi}^{1}: 0 \leq \xi<\xi_{1}\right\}$ of $\left(B^{*}, w^{*}\right)$, and for every element $U \in \mathfrak{U}^{1}$ we get $s_{U} \geq 0, e_{U} \in E$ with $\left\|e_{U}\right\|=1$ and an equivalent norm $p_{U}$ on $E$.

If we have constructed the relatively open partitionings $\mathfrak{U}^{1}, \mathfrak{U}^{2}, \ldots, \mathfrak{U}^{n}$, we consider a fixed element $U_{\xi}^{n}$ of $\mathfrak{U}^{n}=\left\{U_{\xi}^{n}: 0 \leq \xi<\xi_{n}\right\}$. Then we apply the basic step to $U_{\xi}^{n}$ with $p=p_{U_{\xi}^{n}}, \varepsilon=\varepsilon_{n+1}$, and $\beta=\beta_{n+1}$. So we get a partitioning $\mathfrak{U}_{\xi}^{n}=\left\{U_{\xi \eta}^{n}: 0 \leq \eta<\eta_{\xi}^{n}\right\}^{\xi}$ of $U_{\xi}^{n}$ to every element of which a nonnegative

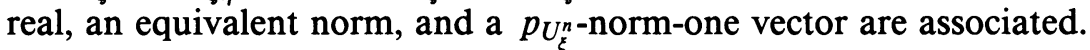

Now $\mathfrak{U}^{n+1}=\bigcup\left\{\mathfrak{U}_{\xi}^{n}: 0 \leq \xi<\xi_{n}\right\}$ is a relatively open partitioning of $B^{*}$ if its elements $U_{\xi \eta}^{n}, 0 \leq \xi<\xi_{n}, 0 \leq \eta<\eta_{\xi}^{n}$, are ordered lexicographically (see [5, Proposition 1.5]). Obviously $\mathfrak{U}^{n+1}$ is a refinement of $\mathfrak{U}^{n}$.

Step 4 . Proof that $\mathfrak{U}$ is separating. Let us assume the contrary, i.e., there exist two different points $x_{1}^{*}$ and $x_{2}^{*}$ in $B^{*}$ such that they belong to one and the same element $V_{n}$ of $\mathfrak{U}^{n}$ for every positive integer $n$. Let us denote by $s_{n}$, $p_{n}$, and $e_{n}$ respectively the nonnegative real number, the equivalent norm, and the unit vector associated to $V_{n}$ by the construction.

Our assumption implies that neither $V_{n}$ is empty nor $V_{n}=\{0\}$ and hence $s_{n}>0$ for every $n \in \mathbb{N}$. The construction gives us that the following properties hold:

(i) $B^{*}=V_{0} \supset V_{1} \supset V_{2} \supset \cdots \supset \bigcap_{n=1}^{\infty} V_{n} \supset\left\{x_{1}^{*}, x_{2}^{*}\right\}$;

(ii) $V_{n} \subset\left\{x^{*} \in V_{n-1}:\left\langle x^{*}, e_{n}\right\rangle>\left(1-\varepsilon_{n}\right) s_{n}\right\}$ for every $n \geq 1$;

(iii) $s_{n}=\sup \left\{p_{n-1}^{*}\left(x^{*}\right): x^{*} \in V_{n}\right\}>0$;

(iv) $p_{n-1}\left(e_{n}\right)=1$ for every $n \geq 1$;

(v) $p_{0}=\|\cdot\|, p_{n}^{2}=p_{n-1}^{2}+\beta_{n} \cdot q_{n}^{2}$, where $q_{n}(x)=\inf \left\{\left\|x-\lambda e_{n}\right\|: \lambda \in \mathbb{R}\right\}$ for every $x \in E$.

Further the proof is contained in [PPhN] (the last half of the proof of Theorem 1.6) with the same notation except for the indexation of the smooth norms which is moved back by one.

1.2. Remark. Theorem 2.2 of [PPhN] states that the dual $\left(E^{*}, w^{*}\right)$ of a smooth space $E$ belongs to the Stegall's class $S$, i.e., every minimal usco correspondence from a Baire space into it is single-valued on a residual subset of its 
domain. (See the definition of "minimal usco" below. For additional information about $S$ see for example [S, S1, K, ČK].) Lemma 5.10 from [N] (or [R, Proposition 2.5]) shows that fragmentable spaces are in $S$ and so Theorem 1.1 immediately yields Theorem 2.2 of [PPhN].

\section{2. $\beta$-DIFFERENTIABILITY AND $\beta$-SMALLNESS}

2.1. Definition. Let $X$ and $Y$ be Hausdorff topological spaces and $F: X \rightarrow Y$ be a multivalued map. $F$ is said to be upper semicontinuous at $x_{0} \in X$ if for every open set $V \subset Y$ that contains $F\left(x_{0}\right)$ there exists a neighborhood $U$ of $x_{0}$ such that $F(x) \subset V$ for every $x \in U$. We say that $F$ is upper semicontinuous if it is upper semicontinuous at every point $x \in X$. If, in addition, $F(x)$ is nonempty and compact for every $x$ in $X$, the correspondence $F$ is called usco.

The graphs of all usco correspondences from $X$ into $Y$ can be ordered by inclusion. Minimal elements of this relation are graphs of usco correspondences from $X$ into $Y$, which will be called "minimal usco". For such mappings the following property holds: for every open subset $U$ of $X$ and every open subset $V$ of $Y$ such that $F(U) \cap V \neq \varnothing$ there exists a nonempty open subset $W$ of $U$ with $F(W) \subset V$ (see $[\mathrm{ChK}])$.

2.2. Definition. Let $\beta$ be a family of nonempty bounded subsets $S$ of the Banach space $E$ satisfying:

(a) $\lambda S \in \beta$ whenever $\lambda \in \mathbb{R}$ and $S \in \beta$;

(b) the union of finitely many members of $\beta$ is in $\beta$;

(c) the union of the members of $\beta$ is all of $E$.

We say that a continuous convex function $f$ on an open convex subset $D$ of $E$ is $\beta$-differentiable at $x \in D(f(x+t y)-f(x)) / t$ if for all $S \in \beta$ the limit of the difference quotient $\lim _{t \rightarrow 0}(f(x+t y)-f(x) / t$ exists uniformly for $y$ in $S$. Natural choices for $\beta$ are all finite sets (Gâteaux differentiability), all weakly compact sets (Hadamard differentiability), or all bounded sets (Fréchet differentiability). If the norm is $\beta$-differentiable at every $x \neq 0$, it is said to be $\beta$-smooth.

We say that $U \subset E^{*}$ is a neighborhood of $x^{*} \in E^{*}$ if there exists $S \in \beta$ with $x^{*}+S^{0} \subset U$, where $S^{0}=\left\{z^{*} \in E^{*}:\left\langle z^{*}, z\right\rangle \leq 1\right.$ for all $\left.z \in S\right\}$. It is easy to check that thus we have introduced a linear topology in $E^{*}$. We will call it $\beta$ as well. This topology is closely related to $\beta$-differentiability by the following fact: The subdifferential mapping $\partial f$ of a continuous convex function $f: D \rightarrow \mathbb{R}$ ( $D$ is an open subset of $E)$ is single-valued and $\beta$-upper semicontinuous at the point $x_{0} \in D$ if and only if $f$ is $\beta$-differentiable at $x_{0}$ (see [PPhN, Proposition 3.1]).

We will need the following

2.3. Definition. Let $X$ be a topological space and $\mathfrak{U}=\bigcup_{n=1}^{\infty} \mathfrak{U}^{n}$ be a $\sigma$-relatively open partitioning of $X$. Let $\tau$ be a second topology on $X$. We say that $\mathfrak{U}$ is $\tau$-small if for every $x \in X$ and for every neighborhood $W$ of $x$ there exist a positive integer $n$ and an element $U$ of $\mathfrak{U}^{n}$ with $x \in U \subset W$.

2.4. Remark. Let $\rho$ be a metric on the topological space $X$ and $\tau_{\rho}$ be the topology generated by $\rho$. If $\rho$ fragments $X$ then there exists a separating 
$\tau_{\rho}$-small $\sigma$-relatively open partitioning of $X$. On the other hand, if $X$ admits a separating $\tau_{\rho}$-small $\sigma$-relatively open partitioning, then $\rho$ fragments all compact subsets of $X$.

The following proposition justifies involving the notion of $\tau$-smallness. It is a generalization of [R, Proposition 2.5].

2.5. Proposition. Let $\left(X, \tau_{1}\right)$ be a topological space, $\tau_{2}$ be a second topology on $X$ and suppose $X$ admits a separating $\tau_{2}$-small $\tau_{1}-\sigma$-relatively open partitioning. Then every minimal usco map $\varphi: B \rightarrow\left(X, \tau_{1}\right)$ from a Baire space $B$ into $\left(X, \tau_{1}\right)$ is single-valued and $\tau_{2}$-upper semicontinuous at every point of $a$ residual subset of $B$.

Proof. Let $\mathfrak{U}=\bigcup_{n=1}^{\infty} \mathfrak{U}^{n}, \mathfrak{U}^{n}=\left\{U_{\xi}^{n}: 0 \leq \xi<\xi^{n}\right\}$ be a separating $\tau_{2}$-small $\tau_{1}-\sigma$-relatively open partitioning of $X$. We put $B_{n}=\{b \in B$ : there exist a neighborhood $V$ of $b$ and an element $U$ of $\mathfrak{U}^{n}$ such that $\left.\varphi(V) \subset U\right\}$ for every $n \in \mathbb{N}$. Obviously the $B_{n}$ are open subsets of $B$. We will prove that they are dense. Indeed, let $V$ be an arbitrary nonempty open subset of $B$ and $n$ be a positive integer. We consider the ordinal number

$$
\hat{\xi}=\min \left\{\xi: U_{\xi}^{n} \cap \varphi(V) \neq \varnothing\right\} .
$$

The minimality of $\varphi$ and $\varphi(V) \cap\left(\bigcup_{\eta<\hat{\xi}+1} U_{\eta}^{n}\right) \neq \varnothing, \bigcup_{\eta<\hat{\xi}+1} U_{\eta}^{n}$ open yield the existence of a nonempty open subset $W$ of $V$ with $\varphi(W) \subset \bigcup_{\eta<\hat{\xi}+1} U_{\hat{\xi}}^{n}$. Hence $\varphi(W) \subset U_{\hat{\xi}}^{n}$ because of the choice of $\hat{\xi}$ and so $W \subset B_{n}$, i.e., $B_{n} \cap V \neq \varnothing$.

Now we will prove that $\varphi$ is single-valued and $\tau_{2}$-continuous at every point $b$ in the residual subset $G=\bigcap_{n=1}^{\infty} B_{n}$ of $B$. As $\varphi(b)$ is contained in one element of $\mathfrak{U}^{n}$ for every $n$, no two points from $\varphi(b)$ can be separated by $\mathfrak{U}$. Therefore $\varphi(b)$ is a singleton ( $U$ is separating). Let $U$ be a $\tau_{2}$-open neighborhood of $\varphi(b)$. Then there exist $n$ and $\alpha_{n}<\xi_{n}$ with $\varphi(b) \in U_{\alpha_{n}}^{n} \subset U$ because $\mathfrak{U}$ is $\tau_{2}$-small. But $b \in G \subset B_{n}$, and hence there exists a neighborhood $V$ of $b$ with $\varphi(V) \subset U_{\alpha_{n}}^{n}$. So $\varphi(V) \subset U$ and $\varphi$ is $\tau_{2}$-upper semicontinuous at $b$.

2.6. Corollary. Let $E$ be a Banach space and $\beta$ be a family of its subsets as described in the beginning of this section. If $\left(E^{*}, w^{*}\right)$ admits a separating $\beta$ small $\sigma$-relatively open partitioning, then every continuous convex function on an open subset of $E$ is $\beta$-differentiable on a residual subset of its domain.

One can prove this using [ChK, Proposition 2.5, Lemma 2.5] or [PPhN, Lemma 3.5].

2.7. Theorem. Suppose that the norm in $E$ is $\beta$-smooth. Then $\left(E^{*}, w^{*}\right)$ admits a separating $\beta$-small $\sigma$-relatively open partitioning.

Because of Proposition 2.5, this theorem is a generalization of [PPhN, Theorem 3.4] but its proof is the same. One should skip the beginning of the proof in $[\mathrm{PPhN}]$ and use the subsequent argument to show that the $\sigma$-relatively open partitioning constructed in the proof of Theorem 1.1 is actually $\beta$-small.

Combining the results from this section one can obtain once more the following

2.8. Corollary. Let $E$ be a Banach space whose norm is Fréchet smooth. Then the dual norm fragments the bounded subsets of $\left(E^{*}, w^{*}\right)$. 


\section{ACKNOWLEDGMENT}

The author is very grateful to Professor P. S. Kenderov for his fruitful discussions and encouragement.

\section{REFERENCES}

[A] E. Asplund, Fréchet differentiability of convex functions, Acta Math. 121 (1968), 31-47.

[ČK] M. M. Čoban and P. S. Kenderov, Generic Gâteaux differentiability of convex functionals in $C(T)$ and the topological properties of $T$, Math. and Education in Math. 1986 (Proc. 15th Conf. Union of Bulg. Mathematicians, (Sunny Beach, U. B. M. April 1986), pp. 141-149.

[ChK] H. P. R. Christensen and P. S. Kenderov, Dense strong continuity of mappings and the Radon-Nikodym property, Math. Scand. 54 (1984), 70-78.

[JR] J. E. Jayne and C. A. Rogers, Borel selectors for upper semi-continuous set-valued maps, Acta Math. 155 (1985), 41-79.

[K] P. S. Kenderov, Most of the optimization problems have unique solution, Internat. Ser. Numer. Math., vol. 72, Birkhäuser-Verlag, Basel, 1984, pp. 203-216.

[N] I. Namioka, Radon-Nikodym compact spaces and fragmentability, Mathematika 34 (1987), 258-281.

[PPhN] D. Preiss, R. R. Phelps, and I. Namioka, Smooth Banach spaces, weak Asplund spaces and monotone or usco mappings, Israel J. Math. (to appear).

[Ph] R. R. Phelps, Convex functions, monotone operators and differentiability, Lecture Notes in Math., vol. 1364, Springer-Verlag, Berlin, Heidelberg, and New York, 1989.

[R] N. K. Ribarska, Internal characterization of fragmentable spaces, Mathematika 34 (1987), 243-257.

[R1] _ A note on fragmentability of some topological spaces, C. R. Acad. Bulgare Sci. 43 (1990), 13-15.

[S] C. Stegall, A class of topological spaces and differentiation of functions on Banach spaces, Vorlesungen Fachbereich Math. Univ. Essen, 10, (1983), 63-77, 1983.

[S1] _-, More Gâteaux differentiability spaces, Banach spaces Proceedings, Missouri 1984), Lectures Notes in Math., vol. 1166, Springer-Verlag, Berlin, 1985, pp. 158-168.

Department of Mathematics and Informatics, University of Sofia, A. Ivanov 5, Sofia 1126, BULGARIA 